Abstract P177 Table 1

\begin{tabular}{llll}
\hline${ }^{*}$ Age (years) & & 64.1 SD 12.8 & \\
Male:Female & & $51: 21$ & \\
${ }^{*} \mathrm{FEV}_{1} \%$ predicted & $\mathrm{FEV}_{1}$ (L) & $58 \%(\mathrm{SD} 23 \%)$ & 1.32 (SD 0.66) \\
${ }^{*} \mathrm{FVC} \%$ predicted & $\mathrm{FVC} \mathrm{(L)}$ & $79 \%(S D 23 \%)$ & 2.23 (SD 0.89) \\
\hline${ }^{*}$ mean values & &
\end{tabular}

\section{P178 INFORMATION AND EDUCATION NEEDS OF PATIENTS WITH BRONCHIECTASIS: A QUALITATIVE INVESTIGATION}

doi:10.1136/thoraxjnl-2012-202678.239

${ }^{1} \mathrm{KL}$ M Hester, ${ }^{1} \mathrm{~A}$ De Soyza, ${ }^{2} \mathrm{~T}$ Rapley. ${ }^{1}$ Freeman Hospital, Newcastle upon Tyne, UK; ${ }^{2}$ Newcastle University, Newcastle upon Tyne, UK

Introduction Bronchiectasis is a chronic lung condition, characterised by dilated bronchi, causing breathlessness and chronic productive cough, with intermittent infective exacerbations. Patients often have recurrent, costly hospital admissions, a poorer quality of life, and clinically significant fatigue. ${ }^{1}$ Current estimates suggest there are 26,000 adults with the condition in the UK. There is little patient information currently available. Patients reported in a questionnaire that information improved confidence. ${ }^{2}$ Information and education could improve understanding and empower patients to self-manage. Timely recognition and appropriate management of exacerbations could improve disease stability. Qualitative investigation of patients' needs will focus plans for resource development, ensure a user-driven format and content, and improve patient engagement and benefit.

Aims To identify, explore and understand the information needs within this patient group, describing the issues in detail.

Methods An exploratory qualitative study was conducted. Eight patients ( 6 female, 2 male, median age 66 years, range 33-67) were recruited from an adult bronchiectasis clinic in the North of England. In-depth semi-structured interviews were conducted, audio-recorded and transcribed. Thematic analysis was undertaken to identify common themes.

Results Four main interlinking themes emerged:

1. Information: patients felt available information was lacking and accessible resources were needed. A crucial factor for acceptance was trust, with participants expressing a need for information to originate from a reputable source. The majority preferred a web-based resource.

2. Medical care and support: Patients felt that a specialist clinic provided support, help and information, yet there was a need for accessible information outside this setting.

3. Diagnosis experience: this had a big impact on patients; often little information and support was given. They felt more information at an early stage would help patients to cope with and manage bronchiectasis.

4. Living with bronchiectasis and its impact: information and knowledge seemed to be fundamental to patients learning to live with and manage their condition.

Conclusions These findings suggest patients want more information. With trustworthy resources patients could be better equipped to understand and manage bronchiectasis. Further work is planned to inform development and implementation of a user-driven information resource for patients with bronchiectasis.

\section{References}

1. Hester KLM, Macfarlane JG, Tedd H, Jary H, McAlinden P, Rostron L, Small T, Newton JL, De Soyza A. Fatigue in bronchiectasis. OJM 2012 Mar; 105(3):235-40.

2. Hester KLM, McAlinden P, De Soyza A, Education and information in bronchiectasis: what do patients want? European Respiratory Journal. 2011; 38 supplement 55: P3622.

\section{P179 NEBULISED 7\% HYPERTONIC SALINE AS AN ADJUNCT TO AIRWAY CLEARANCE CAN BE COMMENCED SAFELY IN A SUPERVISED ENVIRONMENT WITHOUT THE NEED FOR A FORMAL BRONCHOCONSTRICTION TRIAL}

doi:10.1136/thoraxjnl-2012-202678.240

${ }^{1} \mathrm{NG}$ Mills, ${ }^{1} \mathrm{JR}$ Willis, ${ }^{2} \mathrm{SP}$ Range, ${ }^{2} \mathrm{CM}$ Ohri. 'Physiotherapy Department, Glenfield Hospital, Leicester, UK; 'Institute for Lung Health, Glenfield Hospital, Leicester, UK

Background Nebulised Hypertonic Saline (HTS) has been shown to increase water content of airway surface liquid, enhance mucociliary clearance, reduce exacerbation frequency and improve quality of life in Cystic Fibrosis (CF) and non-CF Bronchiectasis. Historically HTS was associated with bronchoconstriction, therefore formal first dose bronchoconstriction trials (BCT) were performed. Recent studies have shown better tolerance that improves over up to 10 doses (Elkins 2006). We have observed that a fall in FEV1 $>15 \%$ following nebulised HTS rarely correlates with adverse signs or symptoms, questioning the need for a BCT. This study aimed to assess our use of HTS, its safety and tolerance without BCT, and its positive effects.

Methods A senior physiotherapist completed a proforma for all patients commencing HTS over 6 months. HTS was used for different respiratory conditions to aid mucociliary clearance. All patients were pre-dosed with a bronchodilator, and had a closely supervised $1^{\text {st }}$ test dose, replacing formal BCT.

Results 54 patients aged 19-87 years (mean 54, median 61) commenced 7\% HTS. 35 were inpatients and 19 outpatients. The primary diagnoses were: non-CF Bronchiectasis (20), post thoracic surgery (17), CF (13), Pneumonia (2), long term tracheostomy (1), and COPD with Laryngectomy (1). All patients experienced positive effects, listed in table 1 . HTS prescription was individualised: BD regime (42), TDS (8), ODS (4). 13 patients (24\%) experienced adverse effects - increased cough (13), slight chest tightness (3), a $5 \%$ decrease in oxygen saturations (1). All 13 patients and physiotherapists considered the adverse effects sufficiently mild to continue HTS and they subsequently reduced significantly after up to 5 doses. If no adverse effects were experienced during the test dose, no further adverse effects occurred with continued use.

Conclusion This observational study demonstrates that HTS has positive effects in a wide variety of respiratory conditions. We have replaced BCT with a supervised test dose as it was found to a be a safe and efficient evaluation. Over 6 months HTS was well tolerated, with only mild adverse effects, which reduced over subsequent doses. Importantly, there were no adverse effects in patients who had previously undergone an uneventful test dose.

\section{Abstract P179 Table 1}

\begin{tabular}{lc}
\hline Positive effects of test dose & Number \\
\hline$\uparrow$ Sputum cleared & 32 \\
$\uparrow$ Sputum cleared with $\uparrow$ ease & 21 \\
$\downarrow$ Sputum retention & 3 \\
$\uparrow$ Sp02 & 5 \\
$\uparrow$ Lung volume on CXR & 3 \\
$\downarrow$ suction required & 2 \\
Chest felt clearer between treatments & 2 \\
$\downarrow$ Viscosity sputum & 1 \\
$\downarrow$ Work of breathing & 1 \\
$\downarrow$ Frequency of physiotherapy needed & 1 \\
$\downarrow$ Anxiety with airway clearance & 1 \\
$\uparrow$ Clearance nasal secretions & 1 \\
Facilitated weaning from the ventilator & 1 \\
\hline
\end{tabular}

